\title{
Ubiquitin-proteasome system, a new anti-tumor target
}

\author{
Wei DU ${ }^{1}$, Qi-bing $\mathrm{MEI}^{2}$
}

Acta Pharmacologica Sinica (2013) 34: 187-188; doi: 10.1038/aps.2012.192

U biquitin-proteasome system (UPS) is a major pathway for intracellular protein degradation. Its target proteins include a broad array of regulatory proteins that play important roles in cell cycle progression, cell development and differentiation, DNA damage responses, and tumorgenesis. Dysfunction of UPS gives rise to various kinds of diseases including infectious diseases, autoimmune diseases and cancers.

The ubiquitylation process carries out by three classes of enzymes, E1 (activating enzyme), E2 (conjugating enzyme) and E3 (ubiquitin ligase). The ubiquitin is activated by E1 in an ATP-dependent manner, and then transferred to E2, and finally E3 adds ubiquitin on a target protein covalently as a tag, which can be recognized by proteasome for degradation. The regulation of UPS is rather complicated. Human genome encodes hundreds of E3 ligases super family, which are responsible for specificity of protein degradation. Cullin-RING ligases (CRLs) are the largest family of E3 ubiquitin ligases, which have essential role in controlling numerous cancerrelevant proteins ubiquitylation and proteasomal degradation ${ }^{[1]}$. Therefore, UPS becomes potential drug target for ant-tumor medication.

${ }^{1}$ Department of Immunology, Roswell Park Cancer Institute, Buffalo, NY, USA; ${ }^{2}$ Department of Pharmacology, School of Pharmacy, Fourth Military Medical University, Xi-an 710032, China Correspondence to: Prof Qi-bing MEI (qbmei@fmmu.edu.cn)
The proteasome inhibitor, bortezomib, has been used to treat multiple myeloma and mantle cell lymphoma with great success both clinically and commercially for 5 years. However, as bortezomib targets all ubiquitin-tagged proteins, lacking of substrate specificity may cause many side effects. A series of recently published articles have reported a promising anti-tumor candidate, called MLN4924, which is a small-molecule selective inhibitor of NEDD8-activating enzyme (NAE) ${ }^{[2]}$. NAE, a heterodimer of NAE a and NAE $\beta$, adenylates a small ubiquitin-like protein NEDD8 at its C-terminus in an ATP-dependent manner as the first step in the neddylation cascade, and transfers NEDD8 to UBC12.
Then, NEDD8 is conjugated to cullin protein on CRLs. This covalent modification actives cullin complex to recruit an ubiquitin-charged E2 and leads to downstream target protein polyubiquitination and proteasomal degradation. MLN4924, which can form a covalent adduct with NEDD8, and block NAE at the first step in this pathway, has shown anti-tumor activity in many kinds of cancer cell lines and xenograft models, including lung cancer, colon cancer, melanoma and lymphoma (Figure 1). The anti-tumor activity of MLN4924 results from inactivation of CLRs, which causes DNA re-replication and inhibition of nuclear factor (NF)- $\mathrm{kB}$ signaling, thus leading to cancer cell death ${ }^{[3]}$.

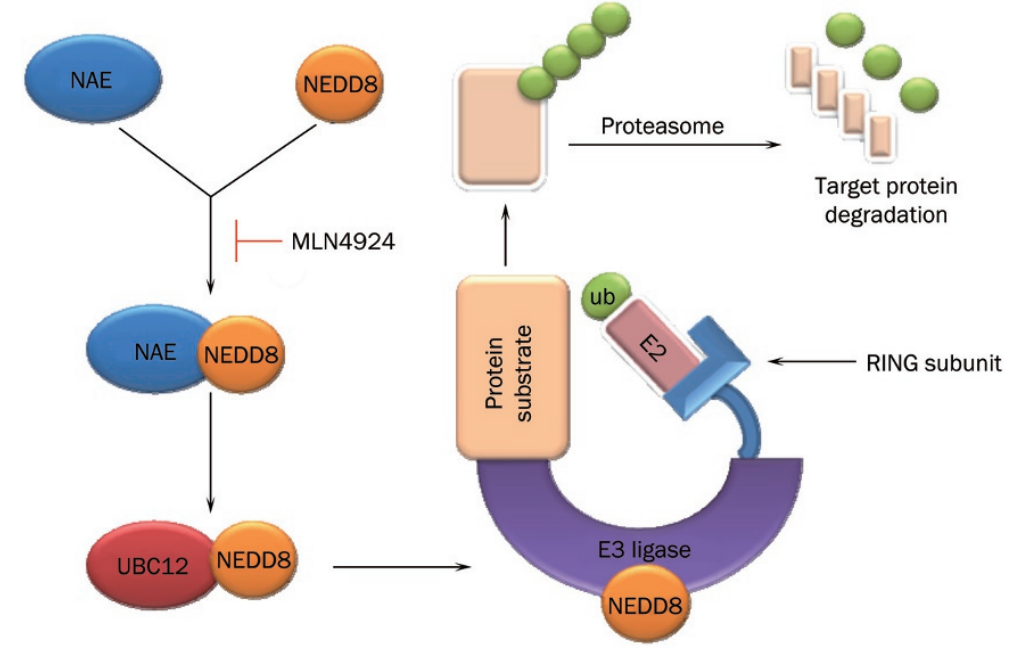

Figure 1. MLN4924 blocks NAE pathway leading to inactivation of CRLs. 
MLN4924 is currently in clinical trials, and its safety for human and clinical activity has been confirmed in both solid tumor and hematologic malignancy. However, the resistance to the agent was also reported in some studies. Early in 2012, researchers found that treatment-emergent heterozygous mutations in the ATP binding pocket and NEDD8-binding cleft of NAE $\beta$ were responsible for the resistance to MLN4924 in cell line and xenograft models of cancer ${ }^{[4]}$. However, the genetic determinants responsible for the sensitivity to MLN4924 are not clear. Further study showed that more than 100 genes contributed to the sensitivity to MLN4924, and approximately a half of them interfered with components of cell cycle, apoptotic machinery, UPS and DNA damage responses. Among them, the genes involved in DNA replication, P53, BRCA1/BRCA2, transcriptioncoupled repair and base excision repair appeared to be important for the lethality of MLN4924. In colon cancer cells, the genes of $\mathrm{G}_{2} / \mathrm{M}$ checkpoint affected the sensitivity to MLN4924. Surprisingly, the p21-dependent intra-S-phase checkpoint and extensive re-replication play an essential role in this process ${ }^{[5]}$.

These investigations on the efficacy of MLN4924 and the genes related to its resistance give us a clearer picture of this potential anti-tumor agent, and fill the gap from bedside to bench work.
1 Emanuele MJ, Elia AE, Xu Q, Thoma CR, Izhar L, Leng Y, et al. Global identification of modular cullinRING ligase substrates. Cell 2011; 147: 459-74.

2 Soucy TA, Smith PG, Milhollen MA, Berger AJ, Gavin JM, Adhikari S, et al. An inhibitor of NEDD8activating enzyme as a new approach to treat cancer. Nature 2009; 458: 732-6.

3 Milhollen MA, Traore T, Adams-Duffy J, Thomas MP, Berger AJ, Dang L, et al. MLN4924, a NEDD8activating enzyme inhibitor, is active in diffuse large B-cell lymphoma models: rationale for treatment of NF-kappaB-dependent lymphoma. Blood 2012; 116: 1515-23.

4 Milhollen MA, Thomas MP, Narayanan U, Traore T, Riceberg J, Amidon BS, et al. Treatmen-emergent mutations in NAE $\beta$ confer resistance to the NEDD8activating enzyme inhibitor MLN4924. Cancer Cell 2012; 21: 388-401.

5 Blank J, Liu XJ, Cosmopoulos K, Bouck DC, Garcia K, Bernard $\mathrm{H}$, et al. Novel DNA damage checkpoints mediating cell death induced by the NEDD8activating enzyme inhibitor MLN4924. Cancer Res 2013; 73: 225-34. 